\title{
Determinantes gestacionais de transtornos mentais em crianças atendidas em hospital pediátrico geral
}

\section{Gestational determinants of mental disorders in children attended in general pediatric hospital}

\author{
Izabel Ester Inácio Ferraz ${ }^{1,2}$. Álvaro Jorge Madeiro Leite². Eugênio de Moura Campos ${ }^{1}$. \\ 1 Hospital Universitário Walter Cantídio (HUWC), Fortaleza, Ceará, Brasil. 2 Universidade Federal do Ceará (UFC), Fortaleza, \\ Ceará, Brasil.
}

\section{RESUMO}

Objetivos: avaliar a associação entre fatores relacionados à história obstétrica materna e surgimento de transtornos mentais em crianças e adolescentes acompanhados em ambulatórios de um hospital infantil. Metodologia: neste estudo transversal, foram aplicados aos cuidadores principais das crianças: termo de consentimento, formulário sociodemográfico contendo possíveis fatores relacionados ao surgimento de transtornos psiquiátricos na infância e Questionário de Capacidades e Dificuldades (SDQ), instrumento principal do estudo. O SDQ é composto por 25 questões divididas em cinco subgrupos de sintomas: comportamento pró social, hiperatividade, sintomas emocionais, problemas de conduta e de relacionamento com colegas. Resultados: após análise bivariada (qui quadrado e anova), concluímos que os fatores relacionados à gestação e nascimento considerados significativos $(\mathrm{p}<0,05)$ para o surgimento futuro de transtornos de saúde mental nas crianças foram: se os pais estavam juntos ao nascimento da criança $(p=0,042)$, se a gestação foi planejada $(p=0,032)$, se a gestação foi desejada $(p=0,038)$, se a mãe utilizou medicamentos na gestação $(\mathrm{p}=0,02)$ e o tempo de amamentação $(\mathrm{p}=0,013)$. Conclusão: concluímos que fatores da história obstétrica e características da depressão materna exercem impacto sobre a saúde mental de crianças pequenas.

Palavras-chave: Crianças. Adolescentes. Psiquiatria.

\begin{abstract}
Objectives: This study aims to evaluate the association between factors related to maternal obstetric history and the emergence of mental disorders in children and adolescents followed at outpatient clinics of a children's hospital. Methodology: In this crosssectional study, it was applied to the primary caregivers of the children: consent form, sociodemographic form containing possible factors related to the emergence of psychiatric disorders in childhood, and Capacities and Difficulties Questionnaire(SDQ), the main instrument of the study. The SDQ is composed of 25 questions divided into five subgroups of symptoms: pro social behavior, hyperactivity, emotional symptoms, behavioral problems and relationships with colleagues. Results: After bivariate (chi-square and anova) analysis, we concluded that the factors related to gestation and birth considered significant $(\mathrm{p}<0.05)$ for future emergence of mental health disorders in children were: if the parents were together at the child's birth $(p=0.042)$, if gestation was desired $(p=0.038)$, if the mother used gestational medications $(p=0.02)$ and the duration of breastfeeding $(p=$ 0.013). Conclusion: We conclude that factors of obstetric history and characteristics of maternal depression have an impact on the mental health of young children.
\end{abstract}

Keywords: Children. Adolescent. Psychiatry.

Autor correspondente: Izabel Ester Inácio Ferraz, Rua Antônio Augusto, 1404, Meireles, Fortaleza, Ceará. CEP: 60110-370. Telefone: +55 85 99676-6207. E-mail: izabeleiferraz@gmail.com

Conflito de interesses: Não há qualquer conflito de interesses por parte de qualquer um dos autores.

Recebido em: 11 Abr 2018; Revisado em: 08 Dez 2018; Aceito em: 08 Dez 2018. 


\section{INTRODUÇÃO}

O papel das relações mãe-filho é fundamental para o desenvolvimento do bebê. Há uma série de influências normativas originadas no ambiente (ou seja, na mãe) dirigidas a esse ser em desenvolvimento, e ações e respostas do bebê. A existência da mãe, sua simples presença, sua menor ação, estimula as respostas do bebê. ${ }^{1}$

Winnicott (2011) considera a mãe construtora ativa no funcionamento mental da criança. Esse autor acreditava que, para existir, o ser humano precisa de atenção e necessita ser cuidado por outro ser humano, e que a mãe suficientemente boa é aquela que possibilita a seu filho a experiência da onipotência primária, fazendo-o crer na ilusão de que o mundo é criado por ele. Localiza, ainda, o início dos problemas psicológicos no vínculo entre recém-nascido e mãe. ${ }^{2}$

Observam-se transtornos mentais em crianças e adolescentes com relativa frequência. As taxas de prevalência dos transtornos na infância vão de 10\% a 20\%. Quando se referem às perturbações mais graves, essas taxas são de $8 \%$ a $10 \%$ da população infantil, o que coloca os transtornos psiquiátricos entre as cinco principais causas de doenças acima de cinco anos de idade. ${ }^{3}$

Cerca de 20 a $40 \%$ das mulheres relatam alguma perturbação emocional ou disfunção cognitiva no período pós-parto. Muitas experimentam a tristeza materna (baby blues), estado normal de tristeza, disforia, choro frequente e dependência, que pode durar vários dias, atribuídos às rápidas alterações hormonais, ao estresse do parto e à conscientização da responsabilidade da maternidade. ${ }^{4,5}$

As depressões pós-parto favorecem o surgimento de distúrbios na criança, desde distúrbios do sono, dificuldades alimentares, ganho insuficiente de peso, problemas dermatológicos, até consultas repetidas ao médico sem motivo. A mãe sente grande cansaço e irritabilidade, que afetam sua relação com o bebê e os cuidados dispensados a ele. Sente incapacidade física para atender às necessidades da criança e falta de prazer ao cuidar dela. A maternagem - todo o cuidado prestado pela mãe ao bebê - torna-se fonte de angústia e pode acarretar maus tratos, até mesmo abusos sexuais. ${ }^{6,7}$

O objetivo deste estudo é avaliar a associação entre fatores relacionados à história obstétrica materna e o surgimento de transtornos mentais em crianças e adolescentes acompanhadas em ambulatórios de pediatria.

\section{MÉTODOS}

Trata-se de estudo transversal, de base hospitalar, em ambulatórios de especialidades pediátricas no Hospital Infantil Albert Sabin, com 307 participantes de 7 a 17 anos, de ambos os sexos. O principal instrumento utilizado foi o Strengths and Difficulties Questionnaire (SDQ, Questionário de Capacidades e Dificuldades), na versão para pais, construído em 1997 por Goodman e validado no Brasil em 2000 por Fleitlich, Cartázar e Goodman. Os dados foram coletados em agosto e setembro de 2015. O SDQ possui 25 questões sobre capacidades e dificuldades, divididas em cinco subgrupos de sintomas psiquiátricos: sintomas emocionais, de conduta, hiperatividade, problemas de relacionamento com colegas e comportamento pró-social. ${ }^{8}$

Cada escala é composta de 5 itens, pontuados como mais ou menos verdadeiro (calculado como 1), ou falso ou verdadeiro (variando conforme o item, podendo pontuar 0 ou 2). A pontuação de cada escala pode variar de 0 a 10 se os 5 itens forem completados. O resultado de cada escala pode ser avaliado se ao menos 3 itens forem completados. A pontuação total de dificuldades é gerada pela soma dos resultados de todas as escalas, exceto a de sociabilidade. $\mathrm{O}$ resultado pode variar de 0 a 40, e os grupos divididos em normal, limítrofe e anormal $^{8}$ (Quadro 1).

Quadro 1. Pontuações das subescalas do SDQ (Strengths and Difficulties Questionnaire).

\begin{tabular}{|l|l|l|l|}
\hline & Normal & Limítrofe & Anormal \\
\hline Pontuação total das dificuldades & $0-13$ & $14-16$ & $17-40$ \\
\hline Pontuação dos sintomas emocionais & $0-3$ & 4 & $5-10$ \\
\hline Pontuação de problemas de conduta & $0-2$ & 3 & $4-10$ \\
\hline Pontuação para hiperatividade & $0-5$ & 6 & $7-10$ \\
\hline Pontuação para problemas com colegas & $0-2$ & 3 & $4-10$ \\
\hline Pontuação para comportamento pró-social & $6-10$ & 5 & $0-4$ \\
\hline
\end{tabular}

Fonte: disponível em: http://www.sdqinfo.com/py/sdqinfo/b3.py?language=Portugueseqz(Brazil). Acesso em: (01 junho 2014).

Em um cenário em que a amostra seja de alto risco, os "casos" podem ser identificados pela pontuação anormal ou limítrofe em uma das quatro escalas de dificuldades; quando a amostra é de baixo risco, os "casos" podem ser identificados pela pontuação anormal em uma das quatro escalas de dificuldades. Neste estudo, nossa amostra é considerada de alto risco por serem crianças com comorbidades clínicas, portanto, chamamos o grupo "limítrofe+anormal" de "casos". 
A variável dependente é definida como "casos". Utilizou-se, além do SDQ, um formulário elaborado pelos autores, com questões sobre variáveis demográficas (cor, idade, escolaridade, situação conjugal dos pais, renda), história obstétrica da mãe (se planejou/desejou a gestação, uso de medicações/drogas, depressão gestacional ou 03 meses após o parto), dados da criança (atividades escolares, de lazer; sono, história patológica), história familiar de doença mental e variáveis sociais (adoção, moradores da casa, abuso sexual, métodos educativos violentos). Estas são variáveis independentes.

Os dados foram organizados em formato padrão, com entrada de dados feita no aplicativo EPI INFO, versão 3.5.1. As medidas de associações foram baseadas em razão de prevalência (RP), com intervalo de confiança de 95\% (IC 95\%). Na análise univariada, investigou-se isoladamente a relação entre cada variável explicativa e a variável dependente (Capacidades e Dificuldades).

A entrevista foi conduzida pela pesquisadora principal e alunos da Faculdade de Medicina da UFC, treinados através de seis seminários teóricos sobre os instrumentos e aplicação prática.

O estudo foi aprovado pelo Comitê de Ética do Hospital Infantil Albert Sabin (número 1.154.890), de acordo com os preceitos contidos na Declaração de Helsinki. Foi assegurada a participação anônima e voluntária pelo Consentimento Livre e Esclarecido antes da realização das entrevistas.

Serão apresentados os resultados quantitativos categóricos em forma de percentuais e contagens, e os numéricos em forma de medidas de tendência central. Foram feitos os testes de $\mathrm{t}$ de Student e chi quadrado para comparação entre variáveis, e consideradas significativas as comparações com valor de $\mathrm{p}<0,05$. Os dados foram tabulados e analisados pelo software SPSS (Statistical Package for the Social Sciences), v23, SPSS Inc. para análise e avaliação dos dados obtidos na coleta.

\section{RESULTADOS}

Do total da amostra (307), 135 crianças foram consideradas casos (44\%), e encontrou-se prevalência de 30,3\% para hiperatividade, 61,6\% para sintomas emocionais, 45,9\% para problemas de conduta, 32,9\% para problemas de relacionamento e $10,1 \%$ para a escala de comportamento pró social não normal.

Em relação aos fatores psicossociais e à prevalência desses transtornos mentais, a análise bivariada encontrou associação estatisticamente significativa $(p<0,05)$ para as seguintes variáveis: depressão materna no período até 03 meses após o nascimento $(\mathrm{p}=0,04)$, doença mental dos pais $(\mathrm{p}=0,04)$ e pais que não vivem juntos atualmente $(\mathrm{p}=0,0002)$.

As mães entrevistadas tiveram, em média, três gestações na vida e dois filhos vivos.

Das mães cujas crianças tiveram seu SDQ com resultado alterado, 29,3\% sofreram aborto, sendo em sua maioria $(91,7 \%)$ espontâneo. Das mães com filhos com SDQ alterado: 10,4\% contam história de filhos falecidos; $81,6 \%$ estavam em união com o pai da criança à época do nascimento desta e tinham em média 24 anos de idade ao engravidar; $66,1 \%$ não planejaram a gestação; 16,9\% não desejaram a gestação, mesmo após saberem que estavam grávidas; 20,2\% tiveram alguma doença na gestação; $22 \%$ utilizaram algum medicamento na gestação; 10,4\% usaram alguma substância ilícita durante a gestação; $8,8 \%$ tiveram depressão na gestação; $1,6 \%$ utilizou psicofármacos durante a gestação; $9,6 \%$ teve depressão no período 03 meses após o parto e 3,2\% usaram psicofármacos nesse período; $64 \%$ tiveram parto normal, a maioria dos filhos nasceu a termo (80,6\%), 92,7\% amamentou e $61,2 \%$ amamentou exclusivamente por pouco mais de 6 meses.

Os fatores relacionados à gestação e nascimento considerados significativos $(\mathrm{p}<0,05)$ para o surgimento futuro de transtornos de saúde mental nas crianças foram: se os pais estavam juntos ao nascimento da criança $(\mathrm{p}=0,042)$, se a gestação foi planejada $(p=0,032)$, se a gestação foi desejada $(p=0,038)$, se a mãe utilizou medicamentos na gestação $(p=0,02)$ e o tempo de amamentação $(p=0,013)$. Não houve diferença estatística para adolescentes.

\section{DISCUSSÃO}

A depressão pós-parto pode ter vários efeitos negativos na mulher e sua criança e particularmente sobre o vínculo mãe-bebê, amamentação e desenvolvimento social, afetivo e cognitivo da criança. $O$ efeito da depressão pós-parto prolongada na criança dura até fases tardias da vida, e está associada a desordens afetivas na infância e adolescência. ${ }^{9}$

$\mathrm{Na}$ depressão materna, a mãe sente tristeza prolongada e sentimento de desesperança. Elas engajam-se menos nas práticas parentais, são menos estimuladoras e menos proativas. A depressão na mãe está associada a problemas sociais e cognitivos nas crianças pequenas desde os primeiros meses de vida e ao longo dos anos pré-escolares. ${ }^{10}$

Quando a mãe recebe ajuda e apoio emocional e é emocionalmente estável, os desafios da parentalidade de crianças pequenas são enfrentados com sucesso. Porém, uma considerável proporção de mulheres jovens em idade reprodutiva apresenta sintomas depressivos graves capazes de comprometer a provisão de cuidados parentais satisfatórios. ${ }^{11}$

Muitos fatores de risco têm sido identificados para a depressão pós-parto. Revisões sugerem que os fatores de risco mais fortes são história de depressão e/ou depressão na gravidez, desvantagem socioeconômica e falta de suporte. Estes fatores de risco parecem aparecer em todas as culturas. ${ }^{9}$

Pesquisas em depressão pós-parto no Brasil identificam fatores de risco similares aos de outros países, com mulheres de classe socioeconômica pobre, alta paridade, não viver com seu parceiro, desordens psicológicas ou psiquiátricas prévias, e gravidez indesejada como maior risco para depressão. ${ }^{9}$ Tais pesquisas são condizentes com os fatores encontrados em nosso estudo. 
Theme e colaboradores, 2016, concluíram que o cuidado com a mãe e o bebê é mais importante na depressão pós-parto do que as intervenções e complicações obstétricas e neonatais. ${ }^{9}$

A depressão materna pode estar associada a episódios depressivos anteriores, pobreza, ser mãe solteira, dificuldades conjugais ou falta ou declínio no apoio social. Complicações gestacionais ou durante o parto, problemas de saúde, irritabilidade e cólicas do recém-nascido podem piorar esses sintomas depressivos nas mulheres. Em casos frequentes, os bebês podem carregar o fardo da depressão da mãe, e se esta vem associada a problemas com o parceiro ou falta de apoio social, o impacto sobre a criança pequena pode ser exacerbado. Algumas crianças pequenas podem, ainda, apresentar maior vulnerabilidade aos efeitos da doença materna do que outras, a depender de seu temperamento, de suas condições de saúde e da presença de um cuidador alternativo. ${ }^{12}$

Pesquisas mostram que mães com depressão crônica são menos sensíveis às demandas de seus bebês, brincando e conversando com suas crianças pequenas com menor frequência, e estabelecendo práticas disciplinares e limites menos adequados à idade do que mães sem depressão. As crianças cujas mães estão deprimidas cronicamente são mais propensas a desenvolver apego não seguro com elas, a mostrar níveis mais baixos de desenvolvimento cognitivo e de linguagem, a revelar-se menos cooperativas e a ter maior dificuldade para controlar raiva e agressividade. ${ }^{13}$

Em 1999, Goodman e Gotlib, propuseram um modelo integrativo para a compreensão dos mecanismos e fatores moderadores que podem estar subjacentes a transmissão de risco para psicopatologia e desenvolvimento anormal em crianças de mães deprimidas. Consideraram quatro mecanismos de transmissão: hereditariedade da depressão, mecanismos neurorreguladores inatos disfuncionais, exposição a cognições, comportamentos e afeto maternos negativos, e o contexto estressante de vida das crianças; além de três fatores mediadores: a saúde do pai e envolvimento com a criança, o curso e o momento de depressão da mãe, e características da criança. ${ }^{14}$

\section{REFERÊNCIAS}

1. Spitz RA. O primeiro ano de vida. São Paulo: Martins Fontes; 2004.

2. Winnicott DW. A família e o desenvolvimento individual. 4. ed. São Paulo: Martins Fontes; 2011.

3. Assumpção FB Jr. Psiquiatria infantil brasileira: um esboço histórico. São Paulo: Lemos Editorial; 1995.

4. Ferraz I. Fatores psicossociais associados ao desenvolvimento de transtornos psiquiátricos em crianças e adolescentes. Rev Med UFC. 2017;57(2):8-13.

5. Sadock BJ, Sadock VA. Compêndio de psiquiatria: ciências do comportamento e psiquiatria clínica. 9. ed. Porto Alegre: Artmed; 2007.

6. Bernardino LM, Fernandes CM (org.). O bebê e a modernidade: abordagens teórico clínicas. São Paulo: Casa do Psicólogo; 2002.
Herba et al, 2013, usando dados de um estudo longitudinal de base populacional de crianças acompanhadas prospectivamente desde os 5 meses até 5 anos, descobriram que as crianças expostas a sintomas depressivos maternos durante o período pré-escolar estavam em risco elevado para sintomas internalizantes (problemas emocionais e de ansiedade), mas que o risco destes problemas foi significativamente reduzido se eles receberam serviços de cuidados infantis precoces. Os benefícios dos serviços de acolhimento para crianças de mães deprimidas existiam independentemente de aquelas terem entrado nos serviços no início da vida (ou seja, antes da idade de 17 meses) ou mais tarde. Mais importante, os resultados para problemas emocionais dependiam dos tipos de cuidados infantis: se regulamentados, baseados em um grupo de cuidados (dentro de um ambiente familiar ou centro), reduziram o risco de problemas emocionais, mas os cuidados prestados por um parente ou babá não o fez. ${ }^{15}$

Sze'kely et al, 2014, mostraram que sintomas depressivos maternos impactam negativamente na capacidade das crianças para identificar verbalmente expressões emocionais, enquanto a sensibilidade materna exerce um efeito benéfico sobre a capacidade das crianças para identificar não verbalmente expressões emocionais. ${ }^{16}$

Os resultados do estudo de Sá et al, 2010, apontaram como fatores estatisticamente relacionados a problemas mentais em crianças e adolescentes: criança/adolescente ser do sexo masculino e sofrer punição física grave; ideação suicida da mãe; violência conjugal física grave contra a mãe; e embriaguez do pai/padrasto. ${ }^{17}$

Crianças pequenas são dependentes de suas mães para estimulação cognitiva e interação social, e, portanto, são mais vulneráveis aos impactos da depressão materna do que crianças em idade escolar ou adolescentes. Essa vulnerabilidade é influenciada, porém, por variáveis relacionadas à família, como suporte familiar que a gestante recebe, e características da depressão materna.

7. Green A. Narcisismo de vida, Narcisismo de morte. Cláudia Berliner, tradutora. São Paulo: Editora Escuta; 1988.

8. Goodman R. Questionário de capacidades e dificuldades (SDQ). [place unknown]: [publisher unknown]; 1999 [Acesso em: 15 maio 2001]. Disponível em: http://www.sdqinfo.com/py/sdqinfo/b3.py?la nguage $=$ Portugueseqz $($ Brazil $)$

9. Theme MM Filha, Ayers S, Gama SG, Leal MC. Factors associated with postpartum depressive symptomatology in Brazil: The Birth in Brazil National Research Study, 2011/2012. J Affective Disord. 2016;194:159-67.

10. Goodman SH. Depression in mothers. Annu Rev Clin Psychol. 2007;3:107-35.

11. Kessler RC. The epidemiology of depression among women. In: Keyes CL, Goodman SH, eds. Women and depression: a handbook 
for the social, behavior, and biomedical sciences. New York: Cambridge University Press; 2006. p. 22-37.

12. Howell EA, Mora PA, DiBonaventura MD, Leventhal H. Modifiable factors associated with changes in postpartum depressive symptoms. Arch Womens Ment Health. 2009;12(2):113-20.

13. NICHD Early Child Care Research Network. Chronicity of maternal depressive symptoms, maternal sensitivity, and child outcomes at 36 months. Dev Psychol. 1999;35(5):1297-310.

14. Goodman SH, Gotlib IH. Risk for psychopathology in the children of depressed mothers: a developmental model for understanding mechanisms of transmission. Psychol Rev. 1999;106(3):458-90.
15. Herba CM, Tremblay RE, Boivin M, Liu X, Mongeau C, Séguin JR, et al. Maternal depressive symptoms and children's emotional problems - can early child care help children of depressed mothers? JAMA Psychiatry. 2013;70(8):830-38.

16. Székely E, Lucassen N, Tiemeier H, Bakermans-Kranenburg MJ, Van Ijzendoorn MH, Kok R, et al. Maternal depressive symptoms and sensitivity are related to young children's facial expression recognition: The Generation R Study. Dev Psychopathol. 2014;26(2):333-45.

17. Sá DG, Bordin IA, Martin D, Paula CS. Fatores de risco para problemas de saúde mental na infância/adolescência. Psic: Teor Pesq. 2010;26(4):643-52.

\section{Como citar:}

Ferraz IE, Leite AJ, Campos EM. Determinantes gestacionais de transtornos mentais em crianças atendidas em hospital pediátrico geral. Rev Med UFC. 2019 jul-set;59(3):39-43. 